\title{
Concepciones de profesores sobre retroalimentación: una revisión de la literatura
}

Conceptions de professeurs par rapport au feedback: une révision de la littérature

Concepções de professores sobre retroalimentação: uma revisão da literatura

Fecha de recepción: 14 DE OCTUBRE DE 2015/Fecha de aceptación: 12 DE OCTUBRE DE 2016/Fecha de disponibilidad en línea: 20 DE FEBRERO DE 2017

Encuentre este artículo en http://magisinvestigacioneducacion.javeriana.edu.co/

\begin{abstract}
Resumen
El artículo presenta una revisión de investigaciones empíricas sobre concepciones de retroalimentación de profesores en distintos niveles educativos. Para ello, se analizaron los resultados de trece estudios que fueron seleccionados entre sesenta y ocho investigaciones publicadas entre 2000 y 2015. Mediante un análisis documental, se establecieron categorías que compilaron aspectos comunes y diferencias en los estudios. Los resultados del análisis permitieron establecer tres tipos de concepciones: corrección, elogio y mejora proyectiva. Se espera que esta revisión contribuya a la comprensión de las prácticas de retroalimentación de profesores y a reducir el déficit de literatura sobre retroalimentación en el mundo hispanoparlante.
\end{abstract}

\section{Palabras clave}

Retroalimentación; investigación; docentes; evaluación

\section{Transferencia a la práctica}

La revisión presentada aporta variadas posibilidades de transferencia a la práctica. En el caso de las prácticas investigativas, puede utilizarse como insumo para promover la realización de estudios sobre retroalimentación en distintos niveles de escolaridad en el contexto hispanoparlante. Respecto de las prácticas educativas, los beneficios descritos sobre retroalimentación proyectiva tienen el potencial de motivar a los profesores a implementar cambios en sus prácticas que generen mejoras sustanciales en los aprendizajes de sus estudiantes. Adicionalmente, podrían permitirles reflexionar sobre los escasos efectos positivos de las fuertemente arraigadas modalidades de retroalimentación como corrección y elogio.

Para citar este artículo / To cite this article / Pour citer cet article / Para citar este artigo

Contreras-Pérez, Gloria \& Zúñiga-González, Carmen Gloria. (2017). Concepciones de profesores sobre retroalimentación: Una revisión de la literatura. magis, Revista Internacional de Investigación en Educación, 9 (19), 69-90. doi: 10.11144/Javeriana.m9-19.cpsr 
Keywords

Feedback; research; teachers; evaluation

Mots clés

Feedback; recherche; enseignants; évaluation

\section{Abstract}

The article presents a review of empirical research on conceptions of feedback of teachers at different levels of education. Accordingly, we analyzed the results of thirteen studies that were selected from sixty-eight research papers published between 2000 and 2015. Based on a documentary analysis, we established categories that compiled common aspects and differences in the studies. Results of the analysis allowed to establish three types of conceptions: correction, praise and projective im provement. We hope that this review wil contribute to the understanding of teacher feedback practices and reduce the deficit of literature about feedback in the Spanish-speaking world.

\section{Transfer to practice}

The review presented offers a range of practical transfer possibilities. In the case of investigative practices, it can be used as an input to promote the conduct of studies on feedback in different levels of schooling in the Spanish-speaking context. Regarding educational practices, the benefits described in projective feedback have the potential to motivate teachers to implement changes in their practices that generate substantial improvements in their Students' learning. In addition, they could allow them to reflect on the limited positive effects of strongly embedded feedback modalities, such as correction and praise.

\section{Transfert à la pratique}

\section{Résumé}

L'article présente une révision de recherches empiriques par rapport aux conceptions de feedback de professeurs de différents niveaux éducatifs. Pour cela, on a analysé les résultats de treize études qui ont étés choisies parmi soixante-dix-huit recherches publiées entre 2000 et 2015. Au moyen d'une analyse documentaire, on a établi les catégories qui réunissent les aspects communs et les différences dans les études. Les résultats de l'analyse nous ont permis d'établir trois types de conceptions: correction, éloge et amélioration prospective. On espère que cette révision contribue à la compréhension des pratiques de feedback de professeurs et à réduire le déficit de littérature sur le feedback dans le monde hispanophone.
La révision présentée apporte une variété de possibilités de transfert à la pratique. Dans le cas des pratiques de recherche, on peut l'utiliser en tant qu'un produit pour encourager la réalisation d'études par rapport au feedback dans les différents niveaux de scolarité dans le contexte hispanophone. Par rapport aux pratiques éducatives, les bénéfices décrits sur le feedback prospectif a le potentiel de motiver aux professeurs à implémenter des changements dans leurs pratiques qui produisent améliorations substantielles dans les apprentissages de leurs étudiants. Par ailleurs, ils pourraient réfléchir sur les rares effets positifs des méthodes fortement enracines de feedback, comme la correction et l'éloge.

\section{Palavras-chave}

Retroalimentação; pesquisa; docentes; avaliação

\section{Resumo}

O artigo apresenta uma revisão de pesquisas empíricas sobre concepções de retroalimentação de professores em diferentes níveis educativos. Para isso, se analisaram os resultados de treze estudos que foram selecionados entre sessenta oito pesquisas publicadas entre 2000 e 2015. Mediante uma análise documental, estabeleceram-se categorias que compilaram aspectos comuns e diferenças nos estudos. Os resultados da análise permitiram estabelecer três tipos de concepções: correção, elogio e melhora projetiva. Espe ra-se que esta revisão contribua ao entendimento das práticas de retroalimentação de professores e a reduzir o déficit de literatura sobre retroalimentação no mundo hispanofalante.

\section{Transferência à prática}

A revisão apresentada gera variadas possibilidades de transferência à prática. No caso das práticas de pesquisa, pode ser utilizada como insumo para promover a realização de estudos sobre retroalimentação em diferentes níveis de escolaridade no contexto hispanofalante. A respeito das práticas educativas, os benefícios descritos sobre retroalimentação projetiva tem o potencial de motivar os professores a implementar mudanças em suas práticas que gerem melhoras substanciais nas aprendizagens de seus estudantes. Adicionalmente, poderiam permitir-lhes refletir sobre os escassos efeitos positivos das fortemente arraigadas modalidades de retroalimentação como correção e elogio. 


\section{Introducción}

Actualmente, un gran número de investigaciones establece la influencia de la evaluación para mejorar los aprendizajes de estudiantes en distintos niveles de formación, incluyendo la universidad. Más específicamente, estos estudios destacan que la retroalimentación para los estudiantes tiene importantes efectos en el monitoreo de sus propios progresos y el desarrollo de habilidades de autorregulación (Biggs \& Tang, 2007; Black \& Wiliam, 1998; Dowden, Pittaway, Yost \& McCarthy, 2013; Entwistle, 2000; Gulikers, Bastiaens, Kirschner \& Kester, 2006; Hattie \& Timperley, 2007; Price, Handley, Millar \& O’Donovan, 2010; Scouller, 1998; Thomson \& Falchikov, 1998). Por lo anterior, este estudio tiene como objetivo analizar concepciones de retroalimentación de los profesores en distintos niveles de educación.

El concepto de retroalimentación se usa en muy diversas disciplinas y de distintas maneras; no obstante, en el campo de la docencia, hay cierto grado de acuerdo en que, para que la retroalimentación tenga efectos positivos, el profesor debe: (a) contar con criterios y estándares de evaluación previamente establecidos y comunicados a los estudiantes; (b) diseñar e implementar un sistema adecuado para recoger la información sobre el aprendizaje, y (c) diseñar estrategias para comunicar esa información y usarla para que los estudiantes aprendan mejor. De acuerdo con esta conceptualización, solo puede hablarse de retroalimentación cuando el proceso lleva a cerrar la brecha entre el actual estado de aprendizaje y el deseado (Black \& Wiliam, 1998; Dowden, Pittaway, Yost \& McCarthy, 2013; Draper, 2009; Havnes, Smith, Dysthe \& Ludvigsen, 2012; Sadler 1989; Wiliam, 2011). Esta distinción se convierte en un elemento clave al momento de revisar concepciones y prácticas de retroalimentación.

En esta revisión, la retroalimentación se considera como parte de la evaluación formativa y, específicamente, se conceptualiza como un proceso de diálogo que el profesor inicia a partir de los resultados de la aplicación de algún procedimiento o actividad de evaluación, que involucra la entrega de comentarios y sugerencias, con el fin de desarrollar en sus estudiantes habilidades de autoevaluación y monitoreo.

En la primera parte de este artículo, se ofrece una breve revisión sobre el concepto de retroalimentación; se esboza una discusión respecto de lo que se entenderá por el constructo "concepciones" y se describe la forma en que los artículos fueron seleccionados y posteriormente analizados. En el apartado referido a la metodología, se analizan los criterios de exclusión e inclusión, las palabras y términos de búsqueda, los buscadores utilizados y, finalmente, las categorías de retroalimentación. En los resultados, se discuten aspectos contextuales de los estudios, sus focos, metodologías usadas y las características de la retroalimentación. En las conclusiones figuran las concepciones predominantes halladas en los estudios y se ofrecen algunas sugerencias para la práctica.

\section{Las concepciones de los profesores}

No es posible comprender la práctica de un profesor en ausencia de sus intenciones, del contexto particular en el que la despliega y de sus dimensiones sociales, históricas y políticas (Carr, 1999). En su sentido más simple, se entiende que una práctica es una puesta en acción que persigue un fin determinado y que se despliega conforme a ciertas reglas. Por otra parte, el sentido de una práctica no es obvio pues se deben comprender las pretensiones que la orientan; es decir, se entiende en referencia a las
Descripción del artículo | Article description | Description de l'article | Artigo descrição

El presente artículo de revisión analiza las concepciones de retroalimentación de profesores en distintos niveles educativos, mediante una revisión de la literatura especializada. Se deriva del proyecto Concepciones de los profesores evaluados sobre retroalimentación del aprendizaje presentes en el portafolios de evaluación docente: diagnóstico y orientaciones para apoyar la docencia de enseñanza media en matemática. 
intenciones del profesional que la desarrolla (Carr, 1999). Finalmente, deben considerarse las circunstancias en las que se desarrolla la acción, de acuerdo a sus planos políticos, sociales e históricos.

El estudio del conjunto de intenciones, pretensiones, fines, teorías y marcos referenciales, entre otros aspectos, como objeto de estudio, se enmarca en el enfoque del pensamiento del profesor, orientado al examen de sus procesos cognitivos y la influencia que estos tienen sobre sus decisiones prácticas. Uno de los elementos más estudiados en este enfoque es el de las concepciones de los profesores. Su importancia radica en el supuesto de que constituyen una valoración o convicción de los profesores sobre algo y actúan como un filtro, por lo cual constituyen un factor que los hace tomar determinadas opciones educativas. Este es el caso de la presente revisión, la cual se relaciona con la retroalimentación que proporcionan a sus alumnos.

Hay consenso al considerar que el pensamiento del profesor está constituido por distintos tipos de saberes o concepciones, adquiridos durante su formación disciplinaria y pedagógica, y a partir de su experiencia y creencias, entre otras fuentes, con mayor influencia de algún tipo de concepción dependiendo en parte del contexto de la acción. Rafael Porlán-Ariza, Ana Rivero-García y Rosa Martín del Pozo (1997) señalan que las concepciones guardan relación más o menos directa con el conocimiento escolar y con su proceso de construcción y facilitación, y se manifiestan de forma más o menos tácita o explícita. Con frecuencia, las concepciones explícitas de los profesores no son coherentes con las implícitas o, dicho de otra forma, la teoría de los profesores no suele ser totalmente coherente con su práctica, de ahí la necesidad de establecer contrastes significativos entre lo explícito y lo implícito (Porlán-Ariza, Rivero-García \& Martín del Pozo, 1997).

Estos autores proponen que el conocimiento profesional dominante (en oposición al conocimiento deseable) es el resultado de la yuxtaposición de cuatro tipos de concepciones, que son de naturaleza diferente, que se generan en distintos momentos y contextos, que se mantienen relativamente aislados unos de otros en la memoria de los profesores y que se manifiestan en diferentes situaciones profesionales o preprofesionales. Estas concepciones son:

1. Los saberes académicos: referidos al conjunto de concepciones o saberes adquiridos fundamentalmente durante la formación profesional inicial. Por esta razón, suelen estar organizados atendiendo a la lógica disciplinar de manera explícita.

2. Los saberes basados en la experiencia: referidos al conjunto de ideas conscientes que los profesores desarrollan en su experiencia. Están enfocados al aprendizaje de los alumnos, la metodología, la evaluación, etc. Son concepciones caracterizadas por un fuerte poder socializador que se comparten habitualmente en el contexto escolar, y que orientan la conducta profesional. No tienen un alto grado de organización interna ya que son saberes adaptativos, a menudo inconsistentes, contradictorios, y cargados de valoraciones morales e ideológicas.

3. Las rutinas y guiones de acción: referidos al conjunto de esquemas tácitos que se originan en el ámbito de lo concreto y que se vinculan más con preguntas del tipo ¿cómo hacerlo? que con preguntas como ipor qué? y ipara qué hacerlo? Contienen pautas de actuación concretas y pertenecen a un tipo de significados que ayudan a resolver una parte importante de la actividad cotidiana, en especial aquella que se repite con cierta frecuencia. 
4. Las teorías implícitas: referidas más bien a un no saber que a un saber, en el sentido de que los profesores no suelen conocer la existencia de estas relaciones entre sus maneras de pensar y actuar y determinadas formalizaciones conceptuales. Este planteamiento es muy similar al que hacen Silvia-Lucía Vilanova, María-del-Mar Mateos-Sanz y María-Basilisa García (2012), quienes destacan que las teorías implícitas son un conjunto de representaciones no conscientes que influye en las formas en que un profesor afronta e interpreta las situaciones en su trabajo. Por ejemplo, un profesor que enseña por transmisión verbal los contenidos disciplinares es muy probable que no sepa, conscientemente, que esta forma de pensar y actuar supone una teoría implícita respecto del alumno: la teoría de la mente en blanco. Son interpretaciones a posteriori acerca de qué teorías dan razón de lo que creemos y de lo que hacemos, aunque lo creamos y hagamos sin saberlo. Estas concepciones no son una construcción de los profesores, sino elaboraciones de los investigadores, que al analizar las prácticas de los profesores, las formulan, es decir, estas representaciones solo pueden ponerse en evidencia con la ayuda de otras personas, como investigadores o colegas. En este tipo de concepciones centra su atención el presente estudio.

En términos metodológicos, las concepciones son difícilmente observables, aunque pueden inferirse por medio de la práctica, como también pueden ser aprehendidas por medio de cuestionarios, escalas, entrevistas colectivas e individuales. No obstante, hay que ser cuidadoso ya que, por lo señalado anteriormente, la explicitación de las concepciones está determinada por una serie de factores y, además, el carácter teórico-práctico del conocimiento profesional puede conducir al profesor a expresar incluso como propias, ideas que no caracterizan su práctica.

\section{Revisiones sobre retroalimentación}

La literatura sobre retroalimentación en idioma español es escasa; el mayor desarrollo de esta área se ha dado en lengua inglesa. Las revisiones disponibles tratan sobre temas específicos como retroalimentación escrita (Agius \& Wilkinson, 2014; Li \& De Luca, 2012), retroalimentación de trabajos en grupo (Gabelica, Van den Bossche, Segers \& Gijselaers, 2102), o retroalimentación en distintos niveles de enseñanza (Evans, 2013). Si bien las revisiones anteriores difieren en el foco de estudio, hay similitudes con respecto a sus postulados sobre concepciones de retroalimentación. Entre ellas hay consenso al indicar que hay diferentes concepciones de retroalimentación, que influyen en el modo en que se perciben las situaciones de aprendizaje y que son difíciles de modificar en el tiempo (Agius \& Wilkinson, 2014; Evans; 2013; Li \& De Luca, 2012). Todas las revisiones indican una carencia de estudios empíricos de retroalimentación en sus múltiples ámbitos, tales como los tipos de retroalimentación y su relación con los diferentes contextos en los que se manifiestan; y sobre creencias, percepciones y concepciones de retroalimentación de profesores y estudiantes en todos los niveles educacionales (Agius \& Wilkinson, 2014; Evans, 2013; Gabelica, Van den Bossche, Segers \& Gijselaers, 2012; Li \& De Luca, 2012).

En esta revisión, la pregunta central es iqué características poseen las concepciones de retroalimentación que orientan las prácticas de los profesores en distintos niveles educativos? 


\section{Metodología}

Para comenzar la búsqueda y recolección de información, primero se revisaron diversas publicaciones y artículos de difusión científica teóricos y prácticos, con el objetivo de definir y caracterizar las concepciones de retroalimentación de los docentes. Una vez realizada la caracterización, esta se utilizó para definir criterios de selección para delimitar la búsqueda y poder descartar aquellos que no se relacionaran directamente con el foco de estudio. Este proceso fue de gran utilidad, pues si bien hay gran cantidad de material relativo a las concepciones de retroalimentación, muchas veces se refiere a las concepciones de estudiantes o directivos.

\section{Criterios de selección}

Los documentos seleccionados para esta revisión debían tener las siguientes características. Respecto del tipo de publicaciones, se buscaron artículos provenientes de revistas arbitradas y capítulos de libros, lo cual implicó dejar fuera tesis de pre y posgrado. Los documentos debían estar escritos en inglés y español. Español por ser el idioma original de las autoras, e inglés por ser un idioma en el cual se publica en extenso acerca de la retroalimentación. Se seleccionaron investigaciones con enfoques cuantitativos, cualitativos y mixtos, publicados entre los años 2000 y 2015. Se excluyeron revisiones, sistematizaciones, análisis documentales o reflexiones. Se consideraron todas las disciplinas presentes en los distintos niveles educacionales. Las temáticas específicas de retroalimentación fueron las relativas a las concepciones de retroalimentación que poseen los profesores en ejercicio y se descartaron las investigaciones sobre prácticas de retroalimentación. Para el caso de las investigaciones que incluían tanto concepciones de profesores como de estudiantes, solo se consideraron los apartados relativos a las concepciones de los docentes.

\section{Palabras de búsqueda y bases de datos}

Las palabras de búsqueda utilizadas se desprendieron de tres conceptos en español e inglés: "concepciones", "profesores" y "prácticas de retroalimentación". En el caso del término "concepciones", se utilizaron también percepciones, creencias, actitudes, conocimiento, comprensión, teoría implícita, marco referencial, conceptions, perceptions, beliefs, attitudes, knowledge, understanding, implicit theory. En la búsqueda del término "profesor", se incluyeron también los conceptos docente, ayudante, tutor, supervisor, maestro; teacher, professor, assistant, tutor, supervisor. Finalmente, al buscar el término "Prácticas de retroalimentación", se utilizaron también las palabras oral, escrita, gestual, grupal, individual, feedback practices, oral, written, non-verbal, group, individual. Todos estos conceptos fueron ingresados en la opción de búsqueda avanzada de las bases de datos y se combinaron de forma aleatoria hasta agotar las posibilidades de búsqueda.

Las bases de datos consultadas fueron las siguientes: A+Education Database; Australian Education Index, AEl; EdITLib, Education \& Information Technology Digital Library; Education Full Text; Education Resources Information Center, ERIC; Education Research Complete; ProQuest Education Journals, y Web of Knowledge.

Luego de realizar la búsqueda en las bases de datos mencionadas, se recolectaron sesenta y ocho artículos, de los cuales cincuenta y cinco fueron descartados porque centraban su atención en concepciones de estudiantes o se trataba de revisiones teóricas y no de investigaciones prácticas. La tabla 1 da cuenta de los trabajos recolectados. 
Tabla 1

Criterios de selección de investigaciones

\begin{tabular}{|c|c|c|}
\hline Criterios & Investigación teórica & Investigación empírica \\
\hline $\begin{array}{l}\text { Concepciones de } \\
\text { los estudiantes }\end{array}$ & $\begin{array}{l}\text { Bonnie Adair-Hauck \& Francis } \\
\text { John Troyan (2013); Thomas J. } \\
\text { Kane \& Douglas O. Staiger (2012) } \\
\text { (total = 2) }\end{array}$ & $\begin{array}{l}\text { Andy Adcroft \& Robert Willis (2013); Doug Atkinson \& Siew } \\
\text { Leng Lim (2013); Glenn Auld, Avis Ridgway \& Judy Williams } \\
\text { (2013); Sebastian Barney, Mahvish Khurum, Kai Petersen, Michael } \\
\text { Unterkalmsteiner \& Ronald Jabangwe (2012); Alasdair Blair, Alun } \\
\text { Wyburn-Powell, Mark Goodwin \& Sam Shields (2014); Sue Bloxham } \\
\text { \& Liz Campbell (2010); Michael A. Buhagiar (2013); Abby Cathcart, } \\
\text { Dominique A. Greer \& Larry Neale (2014); Ching-Huei Chen, Min- } \\
\text { Yu Chung \& Wen-Chi Wu (2013); Andrew D. Cohen \& Marilda C. } \\
\text { Cavalcanti (2009); Sehoya H. Cotner, Bruce A. Fall, Susan M. Wick, } \\
\text { John D. Walker \& Paul M. Baepler (2008); Vasudha Devi, Tatiyana } \\
\text { Mandal, Sunita Kodidela \& Vinod Pallath (2012); David DiBattista, } \\
\text { John O. Mitterer \& Leanne Gosse (2004); Stephen W. Draper } \\
\text { (2009); Cecilia Dube, Sandra Kane \& Miriam Lear (2012); Hendrien } \\
\text { Duijnhouwer, Frans J. Prins \& Karel M. Stokking (2010); Ismail } \\
\text { Ibrahim Elshirbini Abdel Fattah Elashri (2013); Karen Ellery (2008); } \\
\text { Eugenia Etkina (2000); Richard B. Fletcher, Luanna H. Meyer, } \\
\text { Helen Anderson, Patricia Johnston \& Malcolm Rees (2012); Teresa } \\
\text { Guasch, Anna Espasa, Ibis M. Álvarez \& Paul A. Kirschner (2013); } \\
\text { Karen Handley \& Lindsay Williams (2011); Graham D. Hendry, Nikki } \\
\text { Bromberger \& Susan Armstrong (2011); Hui Jiao \& Natalie Brown } \\
\text { (2012); Anastasiya A. Lipnevich, Leigh N. McCallen, Katharine Pace } \\
\text { Miles \& Jeffrey K. Smith (2014); Bunmi S. Malau-Aduli, Dwight } \\
\text { Assenheimer, Derek Choi-Lundberg \& Craig Zimitat (2014); Susan V. } \\
\text { McLaren (2012); Hedy Moscovici (2001); Lene Nordrum, Katherine } \\
\text { Evans \& Magnus Gustafsson (2013); Margaret Price, Karen Handley } \\
\text { \& Jill Millar (2011); Margaret Price, Berry O'Donovan \& Christ Rust } \\
\text { (2007); Carol R. Rodgers (2006); Marieke van der Schaaf, Liesbeth } \\
\text { Baartman, Frans Prins, Anne Oosterbaan \& Haarmen Schaap (2013); } \\
\text { Erna du Toit (2012); Iris Vardi (2012); Jackie Venning \& Femke } \\
\text { Buisman-Pijlman (2013); Darryl A. Wahlstrom (2001); Caroline } \\
\text { Wakefield, James Adie, Edd Pitt \& Tessa Owens (2014); Sylvia Yee } \\
\text { Fan Tang \& Alice Wai Kwan Chow (2007), Binglan Zhou \& Jia Chen } \\
\text { (2010) (total = 40) }\end{array}$ \\
\hline $\begin{array}{l}\text { Concepciones de } \\
\text { los profesores }\end{array}$ & $\begin{array}{l}\text { Stefano Bianchini (2014); David } \\
\text { Boud \& Elizabeth Molloy (2013); } \\
\text { Gary E. Davis \& Mercedes A. } \\
\text { McGowen (2007); Justin C. W. } \\
\text { Debuse, Meredith Lawley \& Rania } \\
\text { Shibl (2008); James J. Kirk, Glenda } \\
\text { Clark-Hayes \& Amy Linsenmayer } \\
\text { (2000); Peter van Petegem \& Jan } \\
\text { Vanhoof (2007); Steve Prowse, } \\
\text { Neil Duncan, Julie Hughes \& } \\
\text { Deirdre Burke (2007); Marie } \\
\text { Annette Vander Kloet \& Brige } \\
\text { Paul Chugh (2012) (total = 8) }\end{array}$ & $\begin{array}{l}\text { Richard Bailey \& Mark Garner (2010); Gavin T. L. Brown, Lois R. } \\
\text { Harris \& Jennifer Harnett (2012); David Carless (2006); Helen } \\
\text { Dixon \& Mavis Haigh (2009); Peter Grainger, Ken Purnell \& Reyna } \\
\text { Zipf (2008); Kerry Harman \& Liz McDowell (2011); Lois Harris, } \\
\text { S. Earl Irving \& Elizabeth Peterson (2008); Mina Jodaie, Tabriz } \\
\text { Branch, Farahman Farrokhi \& Masoud Zoghi (2011); Jinrui Li \& } \\
\text { Roger Barnard (2011); Philip Long (2014); Janice Orrell (2007); Paul } \\
\text { Orsmond \& Stephen Merry (2011); Jinlan Tang \& Colin Harrison } \\
\text { (2011) (total = 13) }\end{array}$ \\
\hline $\begin{array}{l}\text { Prácticas de } \\
\text { retroalimentación }\end{array}$ & & $\begin{array}{l}\text { Nalan Akkuzu (2014); David Carless, Diane Salter, Min Yang \& } \\
\text { Joy Lam (2011); Migchiel van Diggelen, Perry den Brok \& Douwe } \\
\text { Beijaard (2013); Christine Harrison (2013); Carmen Tomas (2014) } \\
\text { (total = 5) }\end{array}$ \\
\hline
\end{tabular}

Fuente: elaboración propia. En sombreado, los artículos seleccionados

\section{Análisis}

Los artículos seleccionados fueron sometidos a un análisis de contenido en dos etapas. Para caracterizar el conjunto de estudios, se hizo primero un breve análisis descriptivo sobre el país en que se realizaron; el nivel de escolaridad involucrado; la forma de conceptualizar la retroalimentación; el foco específico de la retroalimentación en los estudios y las 
metodologías empleadas. Al revisar estos aspectos, emergieron dos categorías más, referidas al constructo asociado a las concepciones en cada estudio y al grado de participación de los sujetos bajo investigación.

En un segundo momento, los artículos fueron sometidos a varias lecturas para caracterizar las concepciones predominantes en el conjunto de estudios. Para este efecto, se usaron las siguientes categorías o clasificaciones de retroalimentación, surgidas de la literatura especializada.

- Negativa-positiva. La retroalimentación se concibe negativa si descalifica o agrede al estudiante, por ejemplo, cuando el profesor considera que su trabajo es de mala calidad. En otro extremo, puede ser positiva, si lo alaba o felicita (Tunstall \& Gipps, 1996).

- Evaluativa-descriptiva. Pat Tunstall y Caroline Gipps (1996) distinguen entre retroalimentación evaluativa que solo juzga el trabajo del estudiante, ya sea en términos positivos o negativos, como castigos y recompensas, y otra descriptiva más bien orientada a describir las fortalezas y debilidades del trabajo.

- Correctiva-orientadora. Una retroalimentación se denomina correctiva si solo señala lo bueno y lo malo del trabajo del estudiante, mientras se denomina orientadora si lo guía a encontrar sus errores y corregirlos por sí mismo (Evans, 2013).

- Específica-general. En relación con el grado de especificidad o detalle, la retroalimentación puede variar dependiendo de factores como la modalidad de formación, la naturaleza de la tarea y de la respuesta del estudiante (Hargreaves, 2013; Shute, 2008).

- Centrada en la tarea-centrada en el proceso-centrada en la autorregulación. Si la retroalimentación tiene como propósito clarificar y reforzar aspectos de la tarea de aprendizaje, se centra en la tarea; si el propósito es orientar al estudiante para seguir adelante con la tarea específica, se denomina de proceso; si se centra en los elementos metacognitivos del estudiante, como el monitoreo y evaluación de las estrategias que utiliza, se denomina de autorregulación (Hattie \& Timperley, 2007).

- Retroactiva-proactiva. Una retroalimentación retroactiva es específica y está orientada a que los estudiantes puedan rehacer la tarea pasada, pudiendo optar a una mejor calificación. La proactiva está más bien centrada en habilidades generales, de manera que puedan tener un mejor desempeño en futuras tareas similares (Jonsson, 2013).

- Convergente-divergente. Una retroalimentación convergente se focaliza en la explicitación de las respuestas correctas y la identificación de errores en el desempeño de los estudiantes; mientras la divergente es exploratoria y estimula a los estudiantes a reconstruir su conocimiento (Torrance \& Pryor, 1998).

La intención en esta etapa fue identificar aquellos aspectos que hablaran sobre cómo los profesores otorgan retroalimentación a sus estudiantes y el sentido que le otorgan, ya sea de manera explícita o implícita.

\section{Resultados}

Las concepciones, entendidas como marcos referenciales que orientan las prácticas de retroalimentación de los profesores, se componen por diferentes tipos de conocimiento y se ven influenciadas por el contexto general y particular en el cual se desempeñan los profesores. El análisis 
destaca, en su primera parte, estos aspectos diferenciadores que están presentes en los estudios revisados, para luego profundizar en los tipos de concepciones encontradas.

La tabla 2 da cuenta de los aspectos generales de cada estudio revisado.

Tabla 2

Aspectos generales de los estudios revisados

\begin{tabular}{|c|c|c|c|}
\hline Autores, año y país & $\begin{array}{l}\text { Tipo de estudio } \\
\text { y técnicas }\end{array}$ & Propósitos & $\begin{array}{l}\text { Nivel de } \\
\text { profesores }\end{array}$ \\
\hline $\begin{array}{l}\text { Richard Bailey \& Mark Garner } \\
\text { (2010), Gran Bretaña. }\end{array}$ & $\begin{array}{l}\text { Cualitativo. } \\
\text { Entrevistas semiestructuradas }\end{array}$ & $\begin{array}{l}\text { Explorar las percepciones sobre la función } \\
\text { y eficacia de la retroalimentación escrita. }\end{array}$ & $\begin{array}{l}\text { Profesores } \\
\text { universitarios. }\end{array}$ \\
\hline $\begin{array}{l}\text { Gavin T. L. Brown, Lois R. } \\
\text { Harris \& Jennifer Harnett } \\
\text { (2012), Nueva Zelanda. }\end{array}$ & $\begin{array}{l}\text { Cuantitativo. Cuestionario } \\
\text { cerrado. }\end{array}$ & $\begin{array}{l}\text { Evaluar las concepciones de } \\
\text { retroalimentación de los profesores de } \\
\text { Nueva Zelanda en el sistema escolar. }\end{array}$ & $\begin{array}{l}\text { Profesores } \\
\text { de primaria y } \\
\text { secundaria. }\end{array}$ \\
\hline David Carless (2006), China. & $\begin{array}{l}\text { Mixto. } \\
\text { Cuestionario con preguntas } \\
\text { cerradas y una pregunta } \\
\text { abierta. Grupos focales. }\end{array}$ & $\begin{array}{l}\text { Examinar la retroalimentación escrita en la } \\
\text { enseñanza universitaria. }\end{array}$ & $\begin{array}{l}\text { Profesores } \\
\text { universitarios. }\end{array}$ \\
\hline $\begin{array}{l}\text { Helen Dixon \& Mavis Haigh } \\
\text { (2009), Nueva Zelanda. }\end{array}$ & $\begin{array}{l}\text { Cualitativo. } \\
\text { Entrevistas semiestructuradas. }\end{array}$ & $\begin{array}{l}\text { Explorar el aprendizaje profesional sobre } \\
\text { retroalimentación de cuatro profesores de } \\
\text { matemáticas, como también los cambios } \\
\text { en su pensamiento. }\end{array}$ & $\begin{array}{l}\text { Profesores } \\
\text { secundarios. }\end{array}$ \\
\hline $\begin{array}{l}\text { Peter Grainger, Ken Purnell \& } \\
\text { Reyna Zipf (2008), Australia. }\end{array}$ & $\begin{array}{l}\text { Cualitativo. } \\
\text { Análisis de documentos. } \\
\text { Entrevistas semiestructuradas. }\end{array}$ & $\begin{array}{l}\text { Reconocer cuáles criterios comunes } \\
\text { utilizan los correctores cuando asignan } \\
\text { calificaciones. Identificar si los correctores } \\
\text { interpretan de la misma forma la "calidad" } \\
\text { medida por estándares. }\end{array}$ & $\begin{array}{l}\text { Profesores } \\
\text { universitarios. }\end{array}$ \\
\hline $\begin{array}{l}\text { Lois Harris, S. Earl Irving \& } \\
\text { Elizabeth Peterson (2008), } \\
\text { Nueva Zelanda. }\end{array}$ & $\begin{array}{l}\text { Cualitativo. } \\
\text { Grupos focales. }\end{array}$ & $\begin{array}{l}\text { Estudiar cómo los profesores entienden la } \\
\text { evaluación y retroalimentación. }\end{array}$ & $\begin{array}{l}\text { Profesores } \\
\text { secundarios. }\end{array}$ \\
\hline $\begin{array}{l}\text { Kerry Harman \& Liz McDowell } \\
\text { (2011), Gran Bretaña. }\end{array}$ & $\begin{array}{l}\text { Cualitativo. } \\
\text { Entrevistas semiestructuradas. }\end{array}$ & $\begin{array}{l}\text { Analizar los recursos discursivos de } \\
\text { profesores al hablar sobre sus experiencias } \\
\text { de evaluación. }\end{array}$ & $\begin{array}{l}\text { Profesores } \\
\text { universitarios. }\end{array}$ \\
\hline $\begin{array}{l}\text { Mina Jodaie, Tabriz Branch, } \\
\text { Farahman Farrokhi \& Masoud } \\
\text { Zoghi (2011), Azerbaiyán. }\end{array}$ & $\begin{array}{l}\text { Mixto. } \\
\text { Cuestionario con preguntas } \\
\text { abiertas y cerradas. } \\
\text { Entrevistas. }\end{array}$ & $\begin{array}{l}\text { Comparar las percepciones de profesores y } \\
\text { estudiantes de segunda lengua, acerca de } \\
\text { la retroalimentación escrita sobre errores } \\
\text { gramaticales. }\end{array}$ & $\begin{array}{l}\text { Profesores y } \\
\text { estudiantes } \\
\text { universitarios. }\end{array}$ \\
\hline $\begin{array}{l}\text { Jinrui Li \& Roger Barnard } \\
\text { (2011), Nueva Zelanda. }\end{array}$ & $\begin{array}{l}\text { Cualitativo. } \\
\text { Grupos focales. Entrevistas } \\
\text { semiestructuradas. Reflexión } \\
\text { en voz alta. }\end{array}$ & $\begin{array}{l}\text { Estudiar las creencias y prácticas de } \\
\text { retroalimentación escrita de tutores sin } \\
\text { experiencia y sin formación pedagógica en } \\
\text { una universidad. }\end{array}$ & $\begin{array}{l}\text { Profesores } \\
\text { universitarios. }\end{array}$ \\
\hline $\begin{array}{l}\text { Paul Orsmond \& Stephen } \\
\text { Merry (2011), Gran Bretaña. }\end{array}$ & $\begin{array}{l}\text { Mixto. } \\
\text { Análisis de pruebas } \\
\text { corregidas. Entrevistas } \\
\text { semiestructuradas. }\end{array}$ & $\begin{array}{l}\text { Estudiar las intenciones de los profesores al } \\
\text { otorgar retroalimentación y la manera en } \\
\text { que es percibida por los estudiantes. }\end{array}$ & $\begin{array}{l}\text { Profesores y } \\
\text { estudiantes } \\
\text { universitarios. }\end{array}$ \\
\hline $\begin{array}{l}\text { Philip Long (2014), Gran } \\
\text { Bretaña. }\end{array}$ & $\begin{array}{l}\text { Cualitativo. } \\
\text { Entrevistas semiestructuradas. }\end{array}$ & $\begin{array}{l}\text { Explorar el concepto de buena } \\
\text { retroalimentación escrita en estudiantes y } \\
\text { sus profesores universitarios. }\end{array}$ & $\begin{array}{l}\text { Profesores y } \\
\text { estudiantes } \\
\text { universitarios. }\end{array}$ \\
\hline Janice Orrell (2007), Australia. & $\begin{array}{l}\text { Cualitativo. } \\
\text { Entrevistas semiestructuradas. } \\
\text { Recurso de "pensamiento } \\
\text { hablado". }\end{array}$ & $\begin{array}{l}\text { Examinar el tipo de retroalimentación que } \\
\text { otorgan profesores a sus estudiantes y, } \\
\text { al respecto, qué mensaje pueden estos } \\
\text { últimos inferir acerca de la evaluación y de } \\
\text { lo que debe ser aprendido. }\end{array}$ & $\begin{array}{l}\text { Profesores } \\
\text { universitarios. }\end{array}$ \\
\hline $\begin{array}{l}\text { Jinlan Tang \& Colin Harrison } \\
\text { (2011), Gran Bretaña. }\end{array}$ & $\begin{array}{l}\text { Mixto. } \\
\text { Cuestionario. Entrevistas en } \\
\text { profundidad. }\end{array}$ & $\begin{array}{l}\text { Explorar cómo un grupo de tutores de in- } \\
\text { glés perciben, interpretan y comprenden los } \\
\text { procesos de retroalimentación. }\end{array}$ & $\begin{array}{l}\text { Profesores uni- } \\
\text { versitarios. }\end{array}$ \\
\hline
\end{tabular}

Fuente: elaboración propia 


\section{Origen de los trabajos}

Todos los trabajos revisados están escritos en inglés. Cinco provienen de Gran Bretaña, tres de Nueva Zelanda, dos de Australia, uno de Azerbaiyán, dos de China.

\section{Nivel de enseñanza de los profesores}

Gavin T. L. Brown, Lois R. Harris y Jennifer Harnett (2012) trabajan con profesores de primaria y secundaria; Helen Dixon y Mavis Haigh (2009) lo hacen con profesores de secundaria; los restantes autores lo hacen con profesores universitarios. Esta distinción es importante pues en general se tiene el panorama de los profesores universitarios.

\section{Sobre el foco de los estudios}

Aunque el propósito macro común a todas las investigaciones es explorar la manera en que los profesores significan la retroalimentación, hay algunas especificidades y matices interesantes de destacar ya que impactan las conclusiones. Por ejemplo, los trabajos de Richard Bailey y Mark Garner (2010), Mina Jodaie, Tabriz Branch, Farahman Farrokhi y Masoud Zoghi (2011), Jinrui Li y Roger Barnard (2011), y Philip Long (2014) centran sus investigaciones en la retroalimentación escrita, mientras que los restantes autores se focalizan en la retroalimentación en sus diversas modalidades.

Por otra parte, las investigaciones abordan la retroalimentación vinculada a evaluaciones con calificación, aunque no todas lo señalan explícitamente. La mayoría de las investigaciones explora las concepciones sobre retroalimentación en general, a excepción de Philip Long (2014) que se focaliza en la retroalimentación de buena calidad. Richard Bailey y Mark Garner (2010) y Paul Orsmond y Stephen Merry (2011) estudian específicamente las concepciones de los profesores sobre las funciones de la retroalimentación. Finalmente, solo el estudio de Helen Dixon y Mavis Haigh (2009) aborda el cambio de concepciones que experimentaron los profesores al participar en el proyecto con investigadores.

\section{Sobre el constructo "concepciones"}

En la revisión efectuada, cinco términos aluden a lo que conocen, piensan o creen los profesores sobre la retroalimentación: concepciones, percepciones, creencias, comprensiones y nociones; este último es el menos usado. En algunos casos, los términos creencias y concepciones se utilizan indistintamente, como en el estudio de Gavin T. L. Brown, Lois R. Harris y Jennifer
Harnett (2012). Una característica común a todos los artículos revisados es que los autores no definen estos términos, aunque, dado el tratamiento tan amplio que le dan, se puede inferir que se trata de marcos referenciales compuestos por diferentes tipos de conocimiento, que orientan las prácticas de retroalimentación de los profesores participantes. Un ejemplo de ello es la investigación de Lois Harris, S. Earl Irving y Elizabeth Peterson (2008), que asocian las concepciones al pensamiento del profesor y otro ejemplo es el estudio de Jinlan Tang y Colin Harrison (2011), que señalan que hay que estudiar las concepciones de los profesores puesto que su percepción de retroalimentación impactará significativamente sus estrategias de retroalimentación, la cantidad de detalle que otorgan, y el tiempo y esfuerzo dedicados a este proceso.

Dado que no se definen, los constructos usados pueden ser interpretados de muy diversas maneras, lo que hace difícil inferir cómo se van construyendo en el tiempo. Puede pensarse que en el caso de los profesores primarios y secundarios, sus concepciones estén en parte constituidas por su formación inicial y continua como profesores, es decir, por conocimiento profesional. En el caso de los docentes universitarios, a quienes no se exige mayor preparación en aspectos pedagógicos (Beaumont, O'Doherty \& Shannon, 2011), probablemente sus concepciones se forman a partir de su experiencia primero como estudiantes de primaria, secundaria y de educación superior, y luego como docentes en la universidad, por lo cual estaríamos más bien en presencia de creencias y teorías implícitas.

\section{Sobre el concepto de retroalimentación}

Se aprecia un tratamiento distinto al concepto de retroalimentación, ya que en la mayoría de los casos se presenta una discusión sobre las diversas formas de definirla, salvo los estudios de Kerry Harman y Liz McDowell (2011), Mina Jodaie, Tabriz Branch, Farahman Farrokhi y Masoud Zoghi (2011), Jinrui Li y Roger Barnard (2011), y Philip Long (2014). En los otros ocho estudios revisados, y dado que la selección se hizo bajo este criterio, las definiciones se relacionan con la evaluación del aprendizaje con los matices que se comentan a continuación.

Janice Orrell (2007) enmarca la retroalimentación como parte de un ciclo ideal que relaciona planificación, enseñanza, aprendizaje, evaluación de aprendizaje y retroalimentación. Citando a John Biggs (1992), Catherine Tang releva el alineamiento constructivo que debe existir entre estos elementos y destaca el rol fundamental que juega la retroalimentación para mejorar los aprendizajes. Lois Harris, S. Earl Irving y Elizabeth Peterson (2008), en uno de sus objetivos 
de investigación, vinculan la retroalimentación que ayuda al aprendizaje específicamente con la evaluación formativa. Helen Dixon y Mavis Haigh (2009), como parte de las políticas públicas en educación en Nueva Zelanda, destacan el fortalecimiento del desarrollo profesional de los profesores del sistema escolar en lo referido al enfoque evaluación para el aprendizaje. En este contexto, la retroalimentación forma parte de este enfoque y tiene como propósito el mejoramiento de los aprendizajes. Jinlan Tang y Colin Harrison (2011) presentan una completa revisión sobre el concepto de retroalimentación y, aunque no ofrecen uno propio, se desprende que la sitúan en el marco de la evaluación formativa. David Carless (2006) la circunscribe a las respuestas a las tareas escritas de los estudiantes (comentarios escritos, anotaciones y marcas de todo tipo, como también comentarios verbales), y destaca que puede tener diversas funciones para el docente, que incluyen justificar una nota, demostrar sapiencia o experiencia, e incluso formar parte del ritual académico.

Dado que el estudio de Peter Grainger, Ken Purnell \& Reyna Zipf (2008) se relaciona con la coherencia de los juicios entre evaluadores, su marco referencial discute la importancia del vínculo entre retroalimentación, criterios y estándares. Gavin T. L. Brown, Lois R. Harris y Jennifer Harnett (2012), en cambio, discuten y adoptan la conceptualización de John Hattie y Helen Timperley (2007), quienes señalan que se trata de información otorgada por algún agente (profesor, par, libro) acerca de aspectos relacionados con el desempeño o comprensión del estudiante.

\section{Sobre el enfoque metodológico empleado}

Gavin T. L. Brown, Lois R. Harris y Jennifer Harnett (2012) presentan el único estudio cuantitativo, con la aplicación masiva de un cuestionario a quinientos diez y ocho profesores del sistema escolar en Nueva Zelanda. Hay ocho estudios cualitativos: Philip Long (2014), con aplicación de entrevistas semiestructuradas a profesores de universidades en Gran Bretaña; Jinrui Li y Roger Barnard (2011) con un estudio de casos en una universidad de Nueva Zelanda con diez y seis profesores; Kerry Harman y Liz McDowell (2011), con entrevistas semiestructuradas a profesores universitarios en Gran Bretaña; Richard Bailey \& Mark Garner (2010) con entrevistas semiestructuradas a profesores universitarios en Gran Bretaña; Helen Dixon y Mavis Haigh (2009) con dos entrevistas semiestructuradas a cuatro profesores de secundaria de matemática en Nueva Zelanda; Lois Harris, S. Earl Irving y Elizabeth Peterson (2008) con grupos focales de profesores secundarios en Nueva Zelanda; y Peter Grainger, Ken Purnell y Reyna Zipf (2008), con entrevistas a cinco profesores universitarios en Australia y Janice Orrell (2007) con entrevistas y aplicación de técnicas de pensamiento hablado y reflexión a profesores universitarios en Australia.

De los restantes estudios, tres presentan una metodología mixta, al aplicar primero un cuestionario - por ejemplo, un inventario o una escala Likert-, para establecer un panorama general o generar criterios de selección de sujetos, y luego dar paso a la etapa cualitativa con entrevistas individuales o grupales. El trabajo de Paul Orsmond y Stephen Merry (2011) constituye la excepción pues aplica una técnica cuantitativa a los comentarios escritos en pruebas y trabajos y, en forma paralela, realiza las entrevistas a los profesores.

El panorama descrito es coherente con la naturaleza de las concepciones ya que difícilmente pueden analizarse mediante un estudio solo cuantitativo, ya que lo que se suele obtener es más bien lo que dicen los profesores. 


\section{Sobre el grado de participación de los profesores}

Dado lo anterior, se estimó pertinente establecer una categoría que evidenciara la relación con el grado de participación de los profesores cuyas concepciones estaban bajo estudio. Si se considera desde el menor hasta el mayor grado de participación, en la investigación de Gavin T. L. Brown, Lois R. Harris y Jennifer Harnett (2012), los profesores solo contestaron un cuestionario cerrado denominado Teachers' Conceptions of Feedback. Esto se reafirma al considerar que el equipo envió los cuestionarios, que luego le fueron devueltos vía postal.

El estudio de Jinlan Tang y Colin Harrison (2011) se inició con la aplicación online de un cuestionario compuesto en su mayoría por preguntas tipo Lickert a cincuenta profesores. Posteriormente, seis de ellos fueron escogidos para la etapa cualitativa en la que participaron con entrevistas en profundidad. Por lo tanto, se aprecia un bajo grado de participación para la mayoría de los profesores participantes. Richard Bailey \& Mark Garner (2010) realizaron entrevistas semiestructuradas a cuarenta y ocho profesores universitarios de distintas carreras de la misma universidad en la que ellos trabajaban. Por el hecho de aplicarse solo una entrevista por separado a cada profesor, se considera que hay un bajo grado de participación. Lois Harris, S. Earl Irving y Elizabeth Peterson (2008) desarrollaron dos grupos focales con once profesores secundarios, que participaban en un proyecto de investigación sobre concepciones de evaluación y retroalimentación. El hecho de haber participado en dos oportunidades en una modalidad grupal les otorga un mayor nivel de involucramiento.

El artículo de Helen Dixon y Mavis Haigh (2009) reporta el cambio de concepciones sobre evaluación y retroalimentación en cuatro profesores de matemática de secundaria, que estaban insertos en un proyecto sobre desarrollo profesional, en el cual tenían que investigar sus propias prácticas, con el apoyo del equipo de investigación y también de sus pares. Dado este contexto, este estudio presenta el mayor grado de participación de los profesores no solo por el hecho de ser ellos investigadores, sino también por el trabajo colaborativo que realizaron.

\section{Sobre las características de la retroalimentación y su sentido}

La tabla 3 muestra las características de la retroalimentación que ofrecen los profesores a sus estudiantes en cada investigación, bien sea captadas mediante la observación directa de sus prácticas, recogidas con algún cuestionario o detalladas en entrevistas. Respecto del sentido, no todos los estudios lo indagan de forma directa, sino que algunos lo infieren a partir de la observación de las prácticas o de lo que declaran los profesores sobre sus prácticas. Por ejemplo, Jinrui Li y Roger Barnard (2011) señalan que, aunque los profesores declaran la finalidad formativa de la retroalimentación, al describir sus prácticas, emerge más bien la finalidad de justificar sus calificaciones. Llama la atención que en cuatro estudios universitarios, los profesores perciben que los estudiantes, en su mayoría, no toman en cuenta la retroalimentación y que solo les interesa la calificación, aunque no ofrezcan mayor evidencia de ello (Carless, 2006; Li \& Barnard, 2011; Long, 2014; Orrell, 2007). Esta visión da forma a sus prácticas e influye en la calidad de retroalimentación que ofrecen, pues deja la idea de que la mejoría del aprendizaje es algo opcional para el estudiante, es decir, de su exclusiva responsabilidad. 
Tabla 3

Características de la retroalimentación ofrecida

\begin{tabular}{|c|c|}
\hline Autores y año & Características de la retroalimentación \\
\hline $\begin{array}{l}\text { Richard Bailey \& Mark } \\
\text { Garner (2010). }\end{array}$ & $\begin{array}{l}\text { La retroalimentación escrita se caracteriza por marcas, puntos y calificaciones. Tiene una } \\
\text { función ambivalente y confusa: es una forma de explicarles a los estudiantes cómo sus trabajos } \\
\text { fueron juzgados, pero también cumple una finalidad administrativa, lo que a veces daña su } \\
\text { valor como apoyo al aprendizaje. En un lugar secundario, aparece la retroalimentación como } \\
\text { motivación, traducida en comentarios positivos de elogio o alabanza. }\end{array}$ \\
\hline $\begin{array}{l}\text { Gavin T. L. Brown, Lois R. } \\
\text { Harris \& Jennifer Harnett } \\
\text { (2012). }\end{array}$ & $\begin{array}{l}\text { Los profesores usan la retroalimentación principalmente para mejorar el trabajo de los } \\
\text { estudiantes y desarrollar su autonomía, más que solo para darles información. Aunque el } \\
\text { elogio o la alabanza aparecen como una forma de retroalimentar, la mayoría de los profesores } \\
\text { no los considera apropiados. }\end{array}$ \\
\hline David Carless (2006). & $\begin{array}{l}\text { Los profesores otorgan calificaciones, marcas, puntajes y comentarios escritos. Aunque esto } \\
\text { puede tener distintas funciones, ellos suponen que a los estudiantes solo les interesa la nota y, } \\
\text { por lo tanto, la función que le otorgan es la de corrección. }\end{array}$ \\
\hline $\begin{array}{l}\text { Helen Dixon \& Mavis Haigh } \\
\text { (2009). }\end{array}$ & $\begin{array}{l}\text { Los profesores van cambiando su concepción de retroalimentación desde una muy básica, } \\
\text { caracterizada por marcas, vistos, cruces y calificaciones, a una comprendida como diálogo } \\
\text { entre profesor y estudiantes que se produce durante la enseñanza y el aprendizaje. }\end{array}$ \\
\hline $\begin{array}{l}\text { Peter Grainger, Ken Purnell } \\
\text { \& Reyna Zipf (2008). }\end{array}$ & $\begin{array}{l}\text { Si bien los profesores comparten la visión sobre los criterios de evaluación que deben usarse } \\
\text { en un instrumento de evaluación en particular, la descripción del estándar no es clara, es } \\
\text { decir, el mismo estudiante puede obtener diferentes resultados de acuerdo a cada evaluador. } \\
\text { Lo anterior redunda en que el tipo de retroalimentación dada es retroactiva y convergente. }\end{array}$ \\
\hline $\begin{array}{l}\text { Lois Harris, S. Earl Irving \& } \\
\text { Elizabeth Peterson (2008). }\end{array}$ & $\begin{array}{l}\text { Emergen tres tipos de retroalimentación. Para el aprendizaje, cuya función es orientar y } \\
\text { mejorar el aprendizaje. Con puntos y calificaciones, con función de informar y cumplir } \\
\text { trámites administrativos. Elogios o alabanzas, cuyo fin es estimular el compromiso con } \\
\text { el estudio. }\end{array}$ \\
\hline $\begin{array}{l}\text { Kerry Harman \& Liz } \\
\text { McDowell (2011). }\end{array}$ & $\begin{array}{l}\text { Los profesores declaran que la evaluación y la retroalimentación deben ser procesos continuos } \\
\text { y horizontales, en los que estudiantes y profesores aprenden de lo ocurrido en las aulas. Sin } \\
\text { embargo, cuando describen sus prácticas de retroalimentación, estas son convergentes, } \\
\text { y centran su atención en si los estudiantes cumplieron o no las metas de aprendizaje } \\
\text { planificadas. }\end{array}$ \\
\hline $\begin{array}{l}\text { Mina Jodaie, Tabriz Branch, } \\
\text { Farahman Farrokhi \& } \\
\text { Masoud Zoghi (2011). }\end{array}$ & $\begin{array}{l}\text { Los profesores perciben que la precisión gramatical es muy importante, por lo tanto, su } \\
\text { retroalimentación está enfocada en corregir errores gramaticales. Se trata de una modalidad } \\
\text { de corrección, pero más bien enfocada en errores y no solo en marcas y calificaciones. }\end{array}$ \\
\hline $\begin{array}{l}\text { Jinrui Li \& Roger Barnard } \\
\text { (2011). }\end{array}$ & $\begin{array}{l}\text { Los profesores declaran que el propósito de la retroalimentación es ayudar a los estudiantes a } \\
\text { mejorar sus habilidades de escritura; sin embargo, en sus prácticas, emerge una preocupación } \\
\text { por informar acerca de cómo se asignaron sus calificaciones y, por lo tanto, hay más bien una } \\
\text { finalidad de justificarlas. }\end{array}$ \\
\hline $\begin{array}{l}\text { Paul Orsmond \& Stephen } \\
\text { Merry (2011). }\end{array}$ & $\begin{array}{l}\text { La categoría de retroalimentación más frecuente fue la alabanza, seguida de identificar o } \\
\text { corregir errores, con tendencia a ser retroactiva y no proactiva. Los investigadores llaman } \\
\text { la atención sobre la poca recurrencia en la categoría "comprometer al estudiante con el } \\
\text { aprendizaje" o "sugerir enfoques para futuros trabajos". }\end{array}$ \\
\hline Philip Long (2014). & $\begin{array}{l}\text { La mayoría del profesorado se centra en la retroalimentación como corrección de errores, que } \\
\text { justifica la calificación. Perciben que la mayoría de los estudiantes simplemente descarta o } \\
\text { desprecia la retroalimentación. }\end{array}$ \\
\hline Janice Orrell (2007). & $\begin{array}{l}\text { Los profesores tienen principalmente una orientación práctica: generar una nota e indicar } \\
\text { errores acerca de la precisión gramatical, conceptual y de ortografía. Hay un mínimo de } \\
\text { compromiso con la construcción de ideas por parte de los estudiantes. }\end{array}$ \\
\hline $\begin{array}{l}\text { Jinlan Tang \& Colin } \\
\text { Harrison (2011). }\end{array}$ & $\begin{array}{l}\text { Emergen tres categorías de retroalimentación. En una, los tutores se caracterizan por ver los } \\
\text { puntajes como lo más relevante y dudan del valor de entregar más detalles; en otra, creen que } \\
\text { es bueno que el tutor ofrezca más que solo vistos y puntos al estudiante para que este pueda } \\
\text { mejorar; en un tercer grupo, los tutores creen que subrayar todos los errores y dar detallada } \\
\text { retroalimentación es lo mejor para los estudiantes. }\end{array}$ \\
\hline
\end{tabular}

Fuente: elaboración propia 


\section{Conclusiones}

El análisis permite establecer que las concepciones de los profesores sobre retroalimentación pueden agruparse en tres tipos. A continuación, se caracteriza cada uno, se discute el sentido otorgado y se comentan algunos aspectos que les confieren especificidad.

\section{Retroalimentación entendida como corrección}

Esta modalidad aparece en especial en la retroalimentación escrita otorgada por docentes universitarios. Se concibe como un proceso en el cual se comunican al estudiante sus aciertos, pero especialmente sus errores, mediante símbolos, marcas, cruces, vistos y puntajes (Bailey \& Garner, 2010; Harris, Irving \& Peterson, 2008; Jodaie, Branch, Farrokhi \& Zoghi, 2011; Li \& Barnard, 2011; Long, 2014; Orsmond \& Merry, 2011; Tang \& Harrison, 2011). Para los profesores, es una manera rápida y clara de identificar los errores, por ejemplo, con subrayados o círculos. En este sentido, se trata de una modalidad más bien correctiva, específica y retroactiva, es decir, identifica los errores cometidos en la tarea pasada. Esta modalidad ha sido denominada por Harry Torrance y John Pryor (1998) como "convergente" y por Mina Jodaie, Tabriz Branch, Farahman Farrokhi y Masoud Zoghi (2011) como "correctiva".

Si se indaga en el sentido que los profesores dan a esta modalidad de retroalimentación, hay dos aspectos interrelacionados. Por una parte, la utilizan para comunicar y explicar a los estudiantes cómo ha sido juzgado y calificado su trabajo, junto con su nivel de desempeño y, por otra, mayoritariamente la usan para justificar la calificación asignada, no solo a sus estudiantes, sino también a sí mismos y a sus superiores.

De acuerdo a la literatura actualizada, este tipo de retroalimentación se considera como evaluativa, puede ser negativa o positiva, y se dirige al ego del estudiante. Las notas, vistos y cruces $u$ otros símbolos otorgan una información muy limitada, por lo cual el estudiante tiene poca posibilidad de mejorar, en especial si la nota no es buena, ya que lo puede desmotivar notablemente (Jonsson, 2013) y afectar su percepción de autoeficacia (Gibbs \& Simpson, 2009). De acuerdo a Royce Sadler (1989), en estos casos, la atención del estudiante se desvía de los criterios y de sus juicios fundamentales derivados, por lo que resulta ser contraproducente para efectos formativos. Los estudios muestran que, aun cuando se ponga una calificación acompañada de comentarios de buena calidad, el estudiante otorga poca atención a mejorar su aprendizaje, ya que está más preocupado de aprobar la asignatura con la calificación obtenida, y se produce un efecto de "cancelación" (Butler, 1988; Crooks, 1988; Gibbs \& Simpson, 2009; Jonsson, 2013; Nicol 2010; Stobart, 2006).

\section{Retroalimentación entendida como elogio}

La otra modalidad utilizada con frecuencia es el elogio o alabanza, retroalimentación evaluativa, positiva y dirigida al ego de los estudiantes, cuya finalidad es promover sentimientos positivos y compromiso con el estudio, en especial en estudiantes a los que les cuesta más obtener buenos resultados (Burnett \& Mandel, 2010; Harris, Irving \& Peterson, 2008; Nelson \& Schunn, 2009). De acuerdo a Gordon Stobart (2006), el elogio en sí no ayuda a mejorar el aprendizaje pues se dirige a la percepción del alumno sobre su persona y no a su desempeño en la tarea retroalimentada. Esto lo conduce a atribuir el éxito en la tarea a su habilidad, la cual percibe como una entidad fija, sin esforzarse para conseguir un aprendizaje exitoso.

Este tipo de retroalimentación, junto con el anteriormente analizado, constituye uno de los más frecuentes en cualquier nivel de escolaridad.

\section{Retroalimentación entendida como mejoría proyectiva}

La otra modalidad de retroalimentación frecuente se caracteriza por comentarios, ya sean orales, escritos o virtuales por medio de alguna plataforma web, que se pueden dar incluso durante el desarrollo de algún trabajo y no solo al final. En este caso, no se encuentra en los artículos mayor detalle acerca del tipo de comentarios que hacen los profesores. No obstante, parece que se realizan mayoritariamente sobre errores, aspectos deficitarios o elementos ausentes en los trabajos de los estudiantes.

El sentido que los profesores le otorgan a esta modalidad es eminente formativo y proyectivo: esperan que los estudiantes mejoren en trabajos a futuro. En este caso, la retroalimentación debe estar más bien centrada en habilidades generales (Jonsson, 2013), y en aquello que necesitan mejorar, más que en cuán bien lo han hecho (Wiliam, 2009).

\section{Consideraciones finales}

Las concepciones recién analizadas se circunscriben a profesores universitarios, ya que los trabajos desarrollados con profesores de primaria y secundaria presentan bastante diferencia con lo discutido. Gavin T. L. Brown, Lois R. Harris y Jennifer Harnett (2012) sugieren que los profesores del sistema escolar en Nueva Zelanda, fuertemente comprometidos con el enfoque de evaluación para el aprendizaje, adoptado desde el 
Ministerio de Educación, sostienen concepciones y prácticas de retroalimentación dirigidas al desarrollo de la autonomía de los estudiantes y al mejoramiento de la calidad de sus trabajos, más que a la corrección de alguna tarea específica o a la autoestima. Por su parte, Helen Dixon y Mavis Haigh (2009), en estudios de casos con cuatro profesores de matemáticas, establecen que los profesores van cambiando su concepción de retroalimentación desde una muy básica, caracterizada por marcas, vistos, cruces y calificaciones, a una comprendida como diálogo entre profesor y estudiantes que se produce durante la enseñanza y el aprendizaje. Por lo tanto, para el caso de los estudios analizados, parece ser que el nivel educacional en el cual se desempeñan los profesores es una variable que influye en la conformación de concepciones sobre retroalimentación. De este modo, se hace necesario seguir indagando en las concepciones de retroalimentación que poseen profesores que han realizado procesos de formación docente -inicial y continua - con la intención de comprender cómo los procesos formativos influyen en el tipo de concepciones que manifiestan.

Por otra parte, en los estudios con profesores que se desempeñan enseñando una segunda lengua (Jodaie, Branch, Farrokhi \& Zoghi, 2011; Tang \& Harrison, 2011), la retroalimentación como corrección es usada de manera preferencial. Esto se comprende dada la naturaleza de la enseñanza y la evaluación en una segunda lengua, ya que se compone en gran parte por aspectos formales y de estructura, lo cual sugiere que el contenido que se evalúa confiere algún tipo de especificidad al tipo de retroalimentación que se otorga y, por lo tanto, a las concepciones que la orientan.

A su vez, como se había anunciado, todos los trabajos se refieren a retroalimentación asociada a procesos de evaluación que derivan en calificación. Esta característica incide en la forma en que los profesores retroalimentan, ya que necesariamente primero deben comunicar al estudiante su nivel de aprendizaje traducido ya sea en un número o concepto, y en la mayoría de los casos acompañado de vistos o cruces. Esta situación, como señalan Christopher J. Harrison, Karen D. Könings, Lambert Schuwirth, Valerie Wass y Cees van der Vleuten (2015), es posible que sea una influencia negativa para la retroalimentación, ya que le confiere una marcada orientación a la corrección. A este respecto, sería interesante indagar en estudios sobre procesos de retroalimentación inmersos en la enseñanza en general, y, en particular, vinculados a la evaluación sin calificación.

Cabe mencionar que cualquier sea el nivel en que se realice la retroalimentación, es importante que haya políticas institucionales que promuevan y normen este proceso, por ejemplo, al promover procesos de formación continua mediante la habilitación de cursos especiales a profesores en todos los niveles escolares.

Pequeños cambios pueden resultar significativos para el aprendizaje de los estudiantes. Al respecto, se recomienda que las acciones de mejora se realicen a inicios de los períodos de clases, porque los estudiantes tienen más tiempo para implementar los cambios sugeridos dentro del semestre. Retroalimentar trabajos de investigación o ensayos ya finalizados sin asignarles calificación, con comentarios individuales a los estudiantes y generales al curso, da la oportunidad a los estudiantes de comprender la naturaleza de sus errores y corregirlos para el trabajo en curso y futuras evaluaciones. Lo anterior, incluso, puede hacerse por los propios estudiantes, al pedirles que comenten el trabajo de sus pares. Además, esta última práctica es coherente con la retroalimentación como diálogo, mejora el autoconcepto de los estudiantes y el clima de aula. Si bien estas acciones pueden resultar una carga adicional para los docentes, por los beneficios percibidos merecen ser implementadas. 
Es menester indicar que esta revisión posee ciertas limitaciones, como la ausencia de estudios analizados sobre el mundo hispanoparlante o la poca representatividad de los estudios seleccionados, al ser ellos en su mayoría de naturaleza cualitativa. Sin embargo, la escasez de estudios realizados en idioma español impidió su inclusión y, a la vez, fue el motor principal para llevar a cabo esta revisión. A su vez, la propia naturaleza de las concepciones indica que estas deben estudiarse cualitativamente, pues solo así es posible desentrañar sus particularidades y complejidad. Por lo anterior, se recomienda que se incremente el número de estudios sobre esta materia en idioma español.

Una última limitación pesquisada es la ausencia de una descripción rica y en profundidad sobre concepciones de retroalimentación ligadas a la mejora proyectiva de los procesos de aprendizaje. Al respecto, este déficit radica en que este tipo de concepción no se manifestó mayormente en los estudios seleccionados. Solo en el caso neozelandés es factible apreciarla, país en que hay una política pública que promueve el enfoque de evaluación para el aprendizaje. De este modo, se considera urgente promover el desarrollo de estudios empíricos sobre concepciones de retroalimentación para poder realizar aquella caracterización y posteriormente difundirla.

\section{Sobre las autoras}

Gloria Contreras-Pérez es profesora de matemáticas y física. Magíster en evaluación educacional y doctora en ciencias de la educación. Académica de la Pontificia Universidad Católica de Valparaíso, PUCV, Chile.

Carmen Gloria Zúñiga-González es licenciada en educación de la Pontificia Universidad Católica de Valparaíso, PUCV, Chile. Doctora en educación de la Universidad de Western Australia, Australia. Profesora asistente de la Universidad Andrés Bello, Chile.

\section{Referencias}

Adair-Hauck, Bonnie \& Troyan, Francis John (2013). A Descriptive and CoConstructive Approach to Integrated Performance Assessment Feedback. Foreign Language Annals, 46 (1), 23-44.

Adcroft, Andy \& Willis, Robert (2013). Do those who benefit the Most Need it the Least? A Four-Year Experiment in Enquiry-Based Feedback. Assessment \& Evaluation in Higher Education, 38 (7), 803-815.

Agius, Natalie M. \& Wilkinson, Ann (2014). Students' and Teachers' Views of Written Feedback at Undergraduate Level: A Literature Review. Nurse Education Today, 34 (4), 552-559. doi:10.1016/j.nedt.2013.07.005

Akkuzu, Nalan (2014). The Role of Different Types of Feedback in the Reciprocal Interaction of Teaching Performance and Self-efficacy Belief. Australian Journal of Teacher Education, 39 (3), 37-66. Disponible en: http://ro.ecu.edu.au/cgi/viewcontent.cgi?article=2207\&context=ajte

Anderson, Kenneth; Benson, Cathy \& Lynch, Tony (2001). Feedback on Writing: Attitudes and Uptake. Edinburgh Working Papers in Applied Linguistics, 11, 1-20. Disponible en: http://files.eric.ed.gov/fulltext/ ED463672.pdf

Atkinson, Doug \& Lim, Siew Leng (2013). Improving Assessment Processes in Higher Education: Student and Teacher Perceptions of the Effectiveness of a Rubric Embedded in a LMS [Learning Management System]. Australasian Journal of Educational Technology, AJET, 29 
(5), 651-666. Disponible en: https://ajet.org.au/ index.php/AJET/article/view/526/755

Auld, Glenn; Ridgway, Avis \& Williams, Judy (2013). Digital Oral Feedback on Written Assignments as Professional Learning for Teacher Educators: A Collaborative Self-Study. Studying Teacher Education, 9 (1), 31-44. Disponible en: https:// www.researchgate.net/publication/262971244_ Digital_Oral_Feedback_on_Written_Assign ments_as_Professional_Learning_for_Teacher_ Educators_A_collaborative_self-study

Bailey, Richard \& Garner, Mark (2010). Is the Feedback in Higher Education Assessment worth the Paper it is Written On? Teachers' Reflections on their Practices. Teaching in Higher Education, 15 (2), 187-198. doi: 10.1080/13562511003620019. Disponible en: https://www.researchgate.net/ publication/232849839_Is the feedback in higher_education_assessment_worth_the_pa per_it_is_written_on_Teachers'_reflections_on_ their_practices

Barney, Sebastian; Khurum, Mahvish; Petersen, Kai; Unterkalmsteiner, Michael \& Jabangwe, Ronald (2012). Improving Students with Rubric-Based Self-Assessment and Oral Feedback. IEEE Transactions on Education, ToE, 55 (3), 319-325.

Beaumont, Chris; O’Doherty, Michelle \& Shannon, Lee (2011). Reconceptualising Assessment Feedback: A Key to Improving Student Learning? Studies in Higher Education, 36 (6), 671-687. doi: 10.1080/03075071003731135. Disponible en: https://blogs.deakin.edu.au/ innovation-in-psychology/wp-content/uploads/ sites/24/2013/11/2011_improving_assessment feedback_dialogic_model.pdf

Bianchini, Stefano (2014). Feedback Effects of Teaching Quality Assessment: Macro and Micro Evidence. Assessment \& Evaluation in Higher Education, 39 (3), 380-394.

Biggs, John \& Tang, Catherine (2007). Teaching for Quality Learning at University. New York: The Society for Research into Higher Education, SRHE \& Open University Press. Disponible en: http://www.umweltbildung-noe.at/upload/files/ OEKOLOG\%202014/2_49657968-Teaching-forQuality-Learning-at-University.pdf

Black, Paul \& Wiliam, Dylan (1998). Assessment and Classroom Learning. Assessment in Education: Principles, Policy \& Practice, 5 (1), 7-74. Disponible en: http://www.tandfonline. com/doi/pdf/10.1080/0969595980050102. Disponible en: https://www.dora.dmu.ac.uk/ handle/2086/10245? show $=$ full

Blair, Alasdair; Wyburn-Powell, Alun; Goodwin, Mark \& Shields, Sam (2014). Can Dialogue
Help to Improve Feedback on Examinations? Studies in Higher Education, 39 (6), 1039-1054. doi: 10.1080/03075079.2013.777404. Disponible en: https://dora.dmu.ac.uk/handle/2086/ 10245? show =full

Bloxham, Sue \& Campbell, Liz (2010). Generating Dialogue in Assessment Feedback: Exploring the Use of Interactive Cover Sheets. Assessment \& Evaluation in Higher Education, 35 (3), 291-300. Disponible en: http://www.library.uwa.edu. au/_data/assets/pdf_file/0005/1888439/Blox ham-2010.pdf

Boud, David \& Molloy, Elizabeth (2013). Rethinking Models of Feedback for Learning: the Challenge of Design. Assessment \& Evaluation in Higher Education, 38 (6), 698-712. doi: 10.1080/02602938.2012.691462. Disponible en: http://www.tandfonline.com/doi/full/10.1080/0 2602938.2012.691462

Brown, Gavin T. L.; Harris, Lois R. \& Harnett, Jennifer (2012). Teacher Beliefs about Feedback within an Assessment for Learning Environment: Endorsement of Improved Learning over Student WellBeing. Teaching and Teacher Education, 28 (7), 968-978. doi:10.1016/j.tate.2012.05.003. Disponible en: http://www.academia.edu/1786470/ Teacher_beliefs_about_feedback_within_an_ Assessment_for_Learning_environment_Endor sement_of_improved_learning_over_student_ well-being

Buhagiar, Michael A. (2013). Mathematics Student Teachers' Views on Tutor Feedback during Teaching Practice. European Journal of Teacher Education, 36 (1), 55-67.

Burnett, Paul C. \& Mandel, Valerie (2010). Praise and Feedback in the Primary Classroom: Teachers' and Students' Perspectives. Australian Journal of Educational \& Developmental Psychology, 10, 145-154.

Butler, Ruth (1988). Enhancing and Undermining Intrinsic Motivation: The Effects of Task-Involving and Ego-Involving Evaluation on Interest and Involvement. British Journal of Educational Psychology, 58 (1), 1-14.

Carless, David (2006). Differing Perceptions in the Feedback Process. Studies in Higher Education, 31 (2), 219-233. doi: 10.1080/03075070600572132. Disponible en: http://www.teachit.so/index_ htm_files/Carless2006.pdf

Carless, David; Salter, Diane; Yang, Min \& Lam, Joy (2011). Developing Sustainable Feedback Practices. Studies in Higher Education, 36 (4), 395407. Disponible en: http://davidcarless.edu.hku. hk/wp-content/uploads/Developing-sustaina ble-feedback-practices.pdf 
Carr, Wilfred (1999). Una teoría para la educación. Hacia una investigación educativa crítica. Madrid: Morata.

Cathcart, Abby; Greer, Dominique A. \& Neale, Larry (2014). Learner-Focused Evaluation Cycles: Facilitating Learning Using Feedforward, Concurrent and Feedback Evaluation. Assessment \& Evaluation in Higher Education, 39 (7), 790802. Disponible en: http://eprints.qut.edu. au/65471/3/65471.pdf

Chen, Ching-Huei; Chung, Min-Yu \& Wu, Wen-Chi (2013). The Effects of Faded Prompts and Feedback on College Students' Reflective Writing Skills. Asia Pacific Education Researcher, 22 (4), 571-583.

Cohen, Andrew D. \& Cavalcanti, Marilda C. (2009). Giving and Getting Feedback on Composition: A Comparison of Teacher and Student Verbal Report. Evaluation \& Research in Education, 1 (2), 63-73. Disponible en: http://anothersample. net/giving-and-getting-feedback-on-compo sition-a-comparison-of-teacher-and-studentverbal-report

Cotner, Sehoya H.; Fall, Bruce A.; Wick, Susan M.; Walker, John D. \& Baepler, Paul M. (2008). Rapid Feedback Assessment Methods: Can We Improve Engagement and Preparation for Exams in Large-Enrollment Courses? Journal of Science Education and Technology, 17 (5), 437-443. Disponible en: http://booksc.org/book/19351946

Crooks, Terence J. (1988). The Impact of Classroom Evaluation Practices on Students. Review of Educational Research, 58 (4), 438-481. Disponible en: http://datause.cse.ucla.edu/DocS/tjc imp_1988.pdf

Davis, Gary E. \& McGowen, Mercedes A. (2007). Formative Feedback and the Mindful Teaching of Mathematics. Australian Senior Mathematics Journal, ASMJ, 21 (1), 19-30. Disponible en: https://www.researchgate.net/publication/ 259196113_Formative_feedback_and_the_mindful_teaching_of mathematics

Debuse, Justin C. W.; Lawley, Meredith \& Shibl, Rania (2008). Educators' Perceptions of Automated Feedback Systems. Australasian Journal of Educational Technology, 24 (4), 374-386. Disponible en: http://citeseerx.ist.psu.edu/viewdoc/downlo ad?doi=10.1.1.572.3812\&rep =rep1\&type $=$ pdf

Devi, Vasudha; Mandal, Tatiyana; Kodidela, Sunita \& Pallath, Vinod (2012). Integrating Students' Reflection-in-Learning and Examination Performance as a Method for Providing Educational Feedback. Journal of Postgraduate Medicine, 58 (4), 270-274. Disponible en: http://www.jpgmonline.com/article.asp?issn=0022-3859; year=
2012; volume $=58 ;$ issue $=4 ;$ spage $=270 ;$ epage $=2$ 74; aulast $=$ Devi

DiBattista, David; Mitterer, John O. \& Gosse, Leanne (2004). Acceptance by Undergraduates of the Immediate Feedback Assessment Technique for Multiple-Choice Testing. Teaching in Higher Education, 9 (1), 17-28.

Diggelen, Migchiel van; Brok, Perry den \& Beijaard, Douwe (2013). Teachers' Use of a Self-Assessment Procedure: the Role of Criteria, Standards, Feedback and Reflection. Teachers and Teaching, 19 (2), 115-134.

Dixon, Helen \& Haigh, Mavis (2009). Changing Mathematics Teachers' Conceptions of Assessment and Feedback. Teacher Development: An International Journal of Teachers' Professional Development, 13 (2), 173-186. doi: 10.1080/13664530903044002

Dowden, Tony; Pittaway, Sharon; Yost, Helen \& McCarthy, Robin (2013). Students' Perceptions of Written Feedback in Teacher Education: Ideally Feedback is a Continuing Two-Way Communication that Encourages Progress. Assessment \& Evaluation in Higher Education, 38 (3), 349-362. doi: 10.1080/02602938.2011.632676

Draper, Stephen W. (2009). What Are Learners Actually Regulating when Given Feedback? British Journal of Educational Technology, BJET, 40 (2), 306-315.

Dube, Cecilia; Kane, Sandra \& Lear, Miriam (2012). The Effectiveness of Students Redrafting Continuous Assessment Tasks: the Pivotal Role of Tutors and Feedback. Perspectives in Education, 30 (3), 50-59.

Duijnhouwer, Hendrien; Prins, Frans J. \& Stokking, Karel M. (2010). Progress Feedback Effects on Students' Writing Mastery Goal, Self-Efficacy Beliefs, and Performance. Educational Research and Evaluation: An International Journal on Theory and Practice, 16 (1), 53-74.

Elashri, Ismail Ibrahim Elshirbini Abdel Fattah (2013). The Impact of the Direct Teacher Feedback Strategy on the EFL [English as a Foreign Language] Secondary Stage Students' Writing Performance. Disponible en: http://eric.ed.gov/?id=ED540631

Ellery, Karen (2008). Assessment for Learning: A Case Study Using Feedback Effectively in an EssayStyle Test. Assessment \& Evaluation in Higher Education, 33 (4), 421-429.

Entwistle, Noel (2000). Promoting Deep Learning through Teaching and Assessment: Conceptual Frameworks and Educational Contexts. Paper presented at the Teaching and Learning Research Programm [TLRP] Conference, Leicester. Disponible en: http://www.etl.tla.ed.ac.uk/docs/ entwistle2000.pdf 
Etkina, Eugenia (2000). Weekly Reports: A Two-Way Feedback Tool. Science Education, 84 (5), 594-605. Disponible en: https://www.researchgate.net/publication/229991267_Weekly_Reports_A_Two-Way_Feedback_Tool

Evans, Carol (2013). Making Sense of Assessment Feedback in Higher Education. Review of Educational Research, 83 (1), 70-120. doi: 10.3102/0034654312474350. Disponible en: http://eprints.soton. ac.uk/373281/1/70.full.pdf

Fletcher, Richard B.; Meyer, Luanna H.; Anderson, Helen; Johnston, Patricia \& Rees, Malcolm (2012). Faculty and Students Conceptions of Assessment in Higher Education. Higher Education, 64 (1), 119-133.

Gabelica, Catherine; Bossche, Piet van den; Segers, Mien \& Gijselaers, Wim (2012). Feedback, a Powerful Lever in Teams: A Review. Educational Research Review, 7, 123-144. doi: 10.1016/j.edurev.2011.11.003

Gibbs, Graham \& Simpson, Claire (2009). Condiciones para una evaluación continuada favorecedora del aprendizaje. Barcelona: Instituto de Ciencias de la Educación de la Universidad de Barcelona, ICE y Ediciones OCTAEDRO, S.L., Colección Cuadernos de Docencia Universitaria 13. Disponible en: http://www.ub.edu/ice/sites/default/files/docs/ qdu/13cuaderno.pdf

Grainger, Peter; Purnell, Ken \& Zipf, Reyna (2008). Judging Quality through Substantive Conversations between Markers. Assessment \& Evaluation in Higher Education, 33 (2), 133-142.

Guasch, Teresa; Espasa, Anna; Álvarez, Ibis M. \& Kirschner, Paul A. (2013). Effects of Feedback on Collaborative Writing in an Online Learning Environment. Distance Education, 34 (3), 324-338.

Gulikers, Judith T. M.; Bastiaens, Theo J.; Kirschner, Paul \& Kester, Liesbeth (2006). Relations between Student Perceptions of Assessment Authenticity, Study Approaches and Learning Outcome. Studies in Educational Evaluation, 32, 381-400. Disponible en: https://www. researchgate.net/publication/40109369_Relations_between_stu dent_perceptions_of_assessment_authenticity_study_approaches_ and_learning_outcome

Handley, Karen \& Williams, Lindsay (2011). From Copying to Learning: Using Exemplars to Engage Students with Assessment Criteria and Feedback. Assessment \& Evaluation in Higher Education, 36 (1), 95-118.

Hargreaves, Eleanore (2013). Inquiring into Children's Experiences of Teacher Feedback: Reconceptualising Assessment for Learning. Oxford Review of Education, 39 (2), 229-246. Disponible en: http://discovery.ucl.ac.uk/1471462/1/Hargreaves_OXFORD\%20REVIEW\%20HAR GREAVES\%202013_template.pdf

Harman, Kerry \& McDowell, Liz (2011). Assessment Talk in Design: the Multiple Purposes of Assessment in Higher Education. Teaching in Higher Education, 16 (1), 41-52. doi: 10.1080/13562517.2010.507309

Harris, Lois; Irving, S. Earl \& Peterson, Elizabeth (2008). Secondary Teachers' Conceptions of the Purpose of Assessment and Feedback. Paper presented to the Australian Association for Research in Education (AARE) Annual Conference December 2008, Brisbane, Australia. Disponible en: http://www.aare.edu.au/data/publications/2008/ har08349.pdf

Harrison, Christine (2013). Collaborative Action Research as a Tool for Generating Formative Feedback on Teachers' Classroom Assessment Practice: the KREST Project. Teachers and Teaching: Theory and Practice, 19 (2), 202-213, doi: 10.1080/13540602.2013.741839 
Harrison, Christopher J.; Könings, Karen D.; Schuwirth, Lambert; Wass, Valerie \& Vleuten, Cees van der (2015). Barriers to the Uptake and Use of Feedback in the Context of Summative Assessment. Advances in Health Sciences Education, 20 (1), 229-245. doi: 10.1007/s10459-014-9524-6

Hattie, John \& Timperley, Helen (2007). The Power of Feedback. Review of Educational Research, 77 (1), 81-112. Disponible en: http://education.qld. gov.au/staff/development/performance/resour ces/readings/power-feedback.pdf

Havnes, Anton; Smith, Kari; Dysthe, Olga \& Ludvigsen, Kristine (2012). Formative Assessment and Feedback: Making Learning Visible. Studies in Educational Evaluation, 38, 21-27.

Hendry, Graham D.; Bromberger, Nikki \& Armstrong, Susan (2011). Constructive Guidance and Feedback for Learning: the Usefulness of Exemplars, Marking Sheets and Different Types of Feedback in a First Year Law Subject. Assessment \& Evaluation in Higher Education, 36 (1), 1-11. Texto disponible en: http://www.library.uwa.edu.au/ data/assets/pdf_file/0007/1888459/Hendry.pdf

Jiao, Hui \& Brown, Natalie (2012). Providing the Right Feedback to the Right Students: Applying an Innovative E-Assessment System in Engineering Education. Proceedings of the $23^{\text {rd }}$ Annual Conference of the Australasian Association for Engineering Education, AAEE, 3-5 December 2012, Swinburne University of Technology, Melbourne, 1-9. Disponible en: http://ecite.utas.edu.au/81982

Jodaie, Mina; Branch, Tabriz; Farrokhi, Farahman \& Zoghi, Masoud (2011). A Comparative Study of EFL [English as a Foreign Language] Teachers' and Intermediate High School Students' Perceptions of Written Corrective Feedback on Grammatical Errors. English Language Teaching, 4 (4), 36-48. doi:10.5539/elt.v4n4p36. Disponible en: http://files.eric.ed.gov/fulltext/EJ1081040.pdf

Jonsson, Anders (2013). Facilitating Productive Use of Feedback in Higher Education. Active Learning in Higher Education, 14 (1), 63-76. Disponible en: https://feedbackesoe.wikispaces.com/file/view/ Jonsson $+2013+$ facilitating + productive + use + of +feedback+in+higher+education.pdf

Kane, Thomas J. \& Staiger, Douglas O. (dirs.) (2012). Gathering Feedback for Teaching: Combining High-Quality Observations with Student Surveys and Achievement Gains. Policy and Practice Brief. MET Project. Seattle, Washington: Bill \& Melinda Gates Foundation. Disponible en: http://eric.ed.gov/?id=ED540960, http://k12education.gatesfoundation.org/wp-content/ uploads/2015/12/MET_Gathering_Feedback_ Practioner_Brief.pdf
Kirk, James J.; Clark-Hayes, Glenda \& Linsenmayer, Amy (2000). A Mini Assessment Center in the Giving and Receiving of Feedback. Washington, D.C.: Education Resources Information Center, ERIC, Clearinghouse. Disponible en: http://files. eric.ed.gov/fulltext/ED437533.pdf

Li, Jinrui \& Barnard, Roger (2011). Academic Tutors' Beliefs about and Practices of Giving Feedback on Students' Written Assignments: A New Zealand Case Study. Assessing Writing, 16, 137-148. Disponible en: https://www.plymouth.ac.uk/ uploads/production/document/path/7/7172/ Academic_beliefs_about_feedback.pdf

Li, Jinrui \& Luca, Rosemary de (2012). Review of Assessment Feedback. Studies in Higher Education, 39 (2), 378-393. doi: 10.1080/03075079.2012.709494

Lipnevich, Anastasiya A.; McCallen, Leigh N.; Miles, Katharine Pace \& Smith, Jeffrey K. (2014). Mind the Gap! Students' Use of Exemplars and Detailed Rubrics as Formative Assessment. Instructional Science, 42 (4), 539-559.

Long, Philip (2014). Staff and Students' Conceptions of Good Written Feedback: Implications for Practice. Practitioner Research in Higher Education Journal, 8 (1), 54-63. Disponible en: http://194.81.189.19/ojs/index.php/prhe/article/ viewFile/168/290

Malau-Aduli, Bunmi S.; Assenheimer, Dwight; ChoiLundberg, Derek \& Zimitat, Craig (2014). Using Computer-Based Technology to Improve Feedback to Staff and Students on MCQ Assessments. Innovations in Education and Teaching International, 51 (5), 510-522.

McLaren, Susan V. (2012). Assessment is for Learning: Supporting Feedback. International Journal of Technology and Design Education, 22 (2), 227-245.

Moscovici, Hedy (2001). What is Necessary to Include in a Science Methods Course for Teachers on Emergency Permits? The Role of the Feedback Portfolio. Proceedings of the Annual Meeting of the Association for the Education of Teachers in Science, Costa Mesa, California, January 1821, 2001, 724-740. Disponible en: http://eric. ed.gov/?id=ED472910, http://files.eric.ed.gov/ fulltext/ED453083.pdf

Nelson, Melissa M. \& Schunn, Christian D. (2009). The Nature of Feedback: How Different Types of Peer Feedback Affect Writing Performance. Instructional Science, 37 (4), 375-401. Disponible en: http://www.Irdc.pitt.edu/schunn/papers/nelsonschunn-is2009.pdf

Nicol, David (2010). From Monologue to Dialogue: Improving Written Feedback Processes in Mass Higher Education. Assessment \& Evaluation in 
Higher Education, 35 (5), 501-517. Disponible en: http://www.westerlycentre.uwa.edu.au/_data/ assets/pdf_file/0006/1888485/Nicol.pdf

Nordrum, Lene; Evans, Katherine \& Gustafsson, Magnus (2013). Comparing Student Learning Experiences of In-Text Commentary and RubricArticulated Feedback: Strategies for Formative Assessment. Assessment \& Evaluation in Higher Education, 38 (8), 919-940.

Orrell, Janice (2007). Feedback on Learning Achievement: Rhetoric and Reality. Teaching in Higher Education, 11 (4), 441-456.

Orsmond, Paul \& Merry, Stephen (2011). Feedback Alignment: Effective and Ineffective links between Tutors' and Students' Understanding of Coursework Feedback. Assessment \& Evaluation in Higher Education, 36 (2), 125-136. doi: 10.1080/02602930903201651

Petegem, Peter van \& Vanhoof, Jan (2007). Towards a Model of Effective School Feedback: School Heads' Points of View. Educational Research and Evaluation: An International Journal on Theory and Practice, 13 (4), 311-325.

Porlán-Ariza, Rafael; Rivero-García, Ana \& Martín del Pozo, Rosa (1997). Conocimiento profesional y epistemología de los profesores I: teoría, métodos e instrumentos. Enseñanza de las Ciencias, 15 (2), 155-171. Disponible en: http://www. raco.cat/index.php/ensenanza/article/viewFi le/21488/93522

Price, Margaret; Handley, Karen \& Millar, Jill (2011). Feedback: Focusing Attention on Engagement. Studies in Higher Education, 36 (8), 879-896. Disponible en: https://blogs.deakin.edu.au/in novation-in-psychology/wp-content/uploads/si tes/24/2013/11/2011_assessment_fback_as_dia logic_process_pdf

Price, Margaret; Handley, Karen; Millar, Jill \& O'Donovan, Berry (2010). Feedback: All That Effort, But What is the Effect? Assessment \& Evaluation in Higher Education, 35 (3), 277-289. Disponible en: http://www.library.uwa.edu. au/_data/assets/pdf_file/0006/1888530/Price. pdf

Price, Margaret; O’Donovan, Berry \& Rust, Christ (2007). Putting a Social-Constructivist Assessment Process Model into Practice: Building the Feedback Loop into the Assessment Process through Peer Review. Innovations in Education and Teaching International, 44 (2), 143-152.

Prowse, Steve; Duncan, Neil; Hughes, Julie \& Burke, Deirdre (2007). '...Do that and I'll Raise Your Grade.' Innovative Module Design and Recursive Feedback. Teaching in Higher Education, 12 (4), 437-445.
Rodgers, Carol R. (2006). Attending to Student Voice: the Impact of Descriptive Feedback on Learning and Teaching. Curriculum Inquiry, 36 (2), 209-237.

Sadler, Royce (1989). Formative Assessment and the Design of Instructional Systems. Instructional Science, 18, 119-144. Disponible en: https:// www.ualberta.ca/-/media/ualberta/centrefor-teaching-and-learning/symposium/lessteaching-more-learning-2009/royce-sadler/ articles/symposiumltmlroyce-sadlerbformativeassessment-and-the-design-of-instructionalsystems.pdf

Schaaf, Marieke van der; Baartman, Liesbeth; Prins, Frans; Oosterbaan, Anne \& Schaap, Haarmen (2013). Feedback Dialogues that Stimulate Students' Reflective Thinking. Scandinavian Journal of Educational Research, 57 (3), 227-245.

Scouller, Karen (1998). The Influence of Assessment Method on Students' Learning Approaches: Multiple Choice Question Examination versus Assignment Essay. Higher Education, 35 (4), 453-472.

Shute, Valerie J. (2008). Focus on Formative Feedback. Review of Educational Research, 78 (1) 153-189. doi: 10.3102/0034654307313795. Disponible en: http://projects.ict.usc.edu/dlxxi/materials/ Sept2009/Research\%20Readings/Shute\%20 2008\%20Focus\%20 on\%20formative\%20fee dback.pdf

Stobart, Gordon (2006). The Validity of Formative Assessment. En John Gardner (ed.). Assessment and Learning, 133-146. London: Sage Publications.

Tang, Jinlan \& Harrison, Colin (2011). Investigating University Tutor Perceptions of Assessment Feedback: Three Types of Tutor Beliefs. Assessment \& Evaluation in Higher Education, 36 (5), 583-604. doi: 10.1080/02602931003632340

Thomson, K. \& Falchikov, Nancy (1998). "Full on Until the Comes Sun Out": the Effects of Assessment on Students Approaches to Studying. Assessment \& Evaluation in Higher Education, 23 (4), 379-390.

Toit, Erna du (2012). Constructive Feedback as a Learning Tool to Enhance Students' Self-Regulation and Performance in Higher Education. Perspectives in Education, 30 (2), 32-40. Disponible en: http://scholar.ufs.ac.za:8080/xmlui/ bitstream/handle/11660/3549/persed_v30_n2_ a5. pdf? sequence $=1$

Tomas, Carmen (2014). Marking and Feedback Provision on Essay-Based Coursework: a Process Perspective. Assessment \& Evaluation in Higher Education, 39 (5), 611-624.

Torrance, Harry \& Pryor, John (1998). Investigating Formative Assessment. Teaching, Learning and Assessment in the Classroom. Buckingham: Open University Press. 
Tunstall, Pat \& Gipps, Caroline (1996). Teacher Feedback to Young Children in Formative Assessment: a Typology. British Educational Journal, 22 (4), 389-404. Disponible en: http://datause.cse.ucla.edu/DOCS/ pt_tea_1996.pdf

Vander Kloet, Marie Annette \& Chugh, Brige Paul (2012). An Interdisciplinary Analysis of Microteaching Evaluation Forms: How Peer Feedback Forms Shape What Constitutes "Good Teaching". Educational Research and Evaluation: An International Journal on Theory and Practice, 18 (6), 597-612, doi: 10.1080/13803611.2012.704171

Vardi, Iris (2012). The Impact of Iterative Writing and Feedback on the Characteristics of Tertiary Students' Written Texts. Teaching in Higher Education, 17 (2), 167-179.

Venning, Jackie \& Buisman-Pijlman, Femke (2013). Integrating Assessment Matrices in Feedback Loops to Promote Research Skill Development in Postgraduate Research Projects. Assessment \& Evaluation in Higher Education, 38 (5), 567-579.

Vilanova, Silvia-Lucía; Mateos-Sanz, María-del-Mar \& García, María-Basilisa (2011). Las concepciones sobre la enseñanza y el aprendizaje en docentes universitarios de ciencias. Revista Iberoamericana en Educación Superior, 2 (3), 53-75. Disponible en: http://www.redalyc.org/ pdf/2991/299124244003.pdf

Wahlstrom, Darryl A. (2001). The Relationship between Goal Orientation and the Calibration of Performance Expectations to Performance Feedback. Paper presented at the Annual Meeting of the American Educational Research Association, Seattle, Washington, April 10-14, 2001. Disponible en: http://eric.ed.gov/?id=ED452362

Wakefield, Caroline; Adie, James; Pitt, Edd \& Owens, Tessa (2014). Feeding forward from summative assessment: the Essay Feedback Checklist as a Learning Tool. Assessment \& Evaluation in Higher Education, 39 (2), 253-262.

Wiliam, Dylan (2009). Una síntesis integradora de la investigación e implicancias para una nueva teoría de la evaluación formativa. Archivos de Ciencias de la Educación, 3 (3), 15-44. Disponible en http://www.cse. edu.uy/sites/www.cse.edu.uy/files/documentos/pr.4080.pdf

Wiliam, Dylan (2011). What is Assessment for Learning? Studies in Educational Evaluation, 37, 3-14. Disponible en: http://www.udir.no/ globalassets/filer/vurdering/vfl/andre-dokumenter/felles/what-is-as sessment-for-learning1.pdf

Yee Fan Tang, Sylvia \& Wai Kwan Chow, Alice (2007). Communicating Feedback in Teaching Practice Supervision in a Learning-Oriented Field Experience Assessment Framework. Teaching and Teacher Education, 23 (7), 1066-1085.

Zhou, Binglan \& Chen, Jia (2010). The Impact of Teacher Feedback on the Long-Term Improvement in the Accuracy of EFL [English as a Foreign Language] Student Writing. Chinese Journal of Applied Linguistics, 33 (2), 18-34. Disponible en: http://www.celea.org.cn/ teic/90/10060802.pdf 